\title{
Study protocol for a randomised pragmatic trial comparing the clinical and cost effectiveness of lithium and quetiapine augmentation in treatment resistant depression (the LQD study)
}

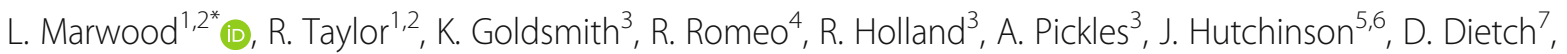 \\ A. Cipriani ${ }^{8,9}$, R. Nair ${ }^{10}$, M.-J. Attenburrow ${ }^{8,9}$, A. H. Young ${ }^{1,2}$, J. Geddes ${ }^{8,9}$, R. H. McAllister-Williams 5,6 \\ and A. J. Cleare ${ }^{1,2}$
}

\begin{abstract}
Background: Approximately 30-50\% of patients with major depressive disorder can be classed as treatment resistant, widely defined as a failure to respond to two or more adequate trials of antidepressants in the current episode. Treatment resistant depression is associated with a poorer prognosis and higher mortality rates. One treatment option is to augment an existing antidepressant with a second agent. Lithium and the atypical antipsychotic quetiapine are two such add-on therapies and are currently recommended as first line options for treatment resistant depression. However, whilst neither treatment has been established as superior to the other in short-term studies, they have yet to be compared head-to-head in longer term studies, or with a superiority design in this patient group.

Methods: The Lithium versus Quetiapine in Depression (LQD) study is a parallel group, multi-centre, pragmatic, openlabel, patient randomised clinical trial designed to address this gap in knowledge. The study will compare the clinical and cost effectiveness of the decision to prescribe lithium or quetiapine add-on therapy to antidepressant medication for patients with treatment resistant depression. Patients will be randomised 1:1 and followed up over 12 months, with the hypothesis being that quetiapine will be superior to lithium. The primary outcomes will be: (1) time to all-cause treatment discontinuation over one year, and (2) self-rated depression symptoms rated weekly for one year via the Quick Inventory of Depressive Symptomatology. Other outcomes will include between group differences in response and remission rates, quality of life, social functioning, cost-effectiveness and the frequency of serious adverse events and side effects.
\end{abstract}

Discussion: The trial aims to help shape the treatment pathway for patients with treatment resistant depression, by determining whether the decision to prescribe quetiapine is superior to lithium. Strengths of the study include its pragmatic superiority design, broad inclusion criteria (external validity) and longer follow up than previous studies.

Trial registration: ISRCTN registry: ISRCTN16387615, registered 28 February 2016. ClinicalTrials.gov: NCT03004521, registered 17 November 2016.

Keywords: Treatment resistant depression, Lithium, Quetiapine, Pragmatic, Randomised clinical trial, Longitudinal, Open-label, Multi-centre, Augmentation, Superiority design

\footnotetext{
* Correspondence: lindsey.marwood@kcl.ac.uk

${ }^{1}$ Centre for Affective Disorders, Department of Psychological Medicine,

Institute of Psychiatry, Psychology \& Neuroscience, King's College London,

London, UK

${ }^{2}$ South London and Maudsley NHS Foundation Trust, London, UK

Full list of author information is available at the end of the article
} 


\section{Background}

Major depressive disorder (MDD) is a highly prevalent and disabling illness requiring effective treatment in order to reduce symptom severity and improve quality of life [1, 2]. Current clinical guidelines recommend the use of antidepressant medication in cases of moderate to severe MDD [3]. However, approximately $30-50 \%$ of patients fail to adequately respond to both first and second line antidepressant treatment trials, and can therefore be described as treatment resistant [3-5]. Existing evidence clearly indicates that the prognoses for patients with treatment resistant MDD (TRD) can be improved with adequate multimodal and/or successive treatment trials $[4,6]$. Given that TRD is associated with a generally poorer prognosis, higher mortality rates and higher healthcare utilisation costs than MDD alone [7, 8], the importance of ensuring that the most effective available medication is fully utilised in clinical practice is clear.

Treatment options for patients with TRD include increasing the dose of a patient's existing antidepressant, switching to another antidepressant (same or different class), or augmenting the existing antidepressant medication with a second agent, for example an additional antidepressant, mood stabiliser, or antipsychotic medication [3]. Increases in dosage have been associated with increased efficacy for some antidepressants [7, 9-11] but not for others [12-15]. Switching to an alternative antidepressant is generally suggested in cases where a patient has either made no response, or is not tolerating their current medication [3], but remission rates to third or fourth line antidepressant treatments are in the order of just 10-15\% [4]. For TRD patients in whom there has been a partial response to current antidepressant treatment, augmentation is usually recommended [3]. However, beyond these general principles, there remains much uncertainty about when to switch and when to augment treatments.

One augmentation strategy is to prescribe a combination of antidepressant medications. However, recent evidence from a large randomised controlled trial indicated that this approach is no more effective than antidepressant monotherapy, questioning its appropriateness [16]. The use of lithium or atypical second-generation antipsychotic augmentation is an approach supported by several recent meta-analyses $[17,18]$. This is reflected in the National Institute of Clinical Excellence (NICE) and the British Association for Psychopharmacology (BAP) treatment guidelines, which emphasise that add-on treatments are a standard treatment pathway for TRD, and include lithium and newer atypical antipsychotics, such as quetiapine, aripiprazole, olanzapine and risperidone, as first line options [3, 19].

Despite these recommendations, few studies have compared the efficacy of lithium versus atypical antipsychotic augmentation head-to-head [3, 20, 21]. The largest and arguably best study to date compared lithium and quetiapine extended release (XR) over just 6 weeks, and found quetiapine to be non-inferior to lithium add-on therapy in patients with TRD [20]. Lithium versus quetiapine augmentation has also been compared in a pilot study of 20 patients, which reported significant reduction in depressive symptoms in both groups, but a greater decrease in Hamilton Depression Rating Scale (HDRS) scores from day 14 onwards in the quetiapine group [21]. However, neither of these comparisons have included a long term follow up, which is needed to ensure that the most effective treatment option is fully utilised in clinical practice, particularly given the chronic nature of TRD, the associated high rates of relapse [8], and the fluctuating nature of depressive symptoms (even in those responding to acute phase treatment) [16]. Clinical guidelines also advise that responders should continue taking the effective medication for at least 6-12 months [3, 19]. Therefore a clinical trial comparing lithium and quetiapine augmentation, with frequent assessment of symptoms and 12 month follow up is clearly warranted.

In the present study, lithium augmentation will comprise one treatment arm, as it is the first-choice add-on treatment for patients with TRD according to the World Federation of Societies of Biological Psychiatry Task Force [22], and a first line treatment option recommended by NICE and BAP [3, 19]. Quetiapine was selected as the atypical antipsychotic comparator, as there is strong evidence for the efficacy of quetiapine augmentation versus placebo [23-25], and it is currently licenced for marketing as an add-on treatment for TRD in the UK in its XR formulation [19]. Although NICE does support the use of other atypical antipsychotics (aripiprazole, risperidone and olanzapine) as add-on treatments in TRD, and there is some evidential support for their use [17, 26], quetiapine is the only atypical antipsychotic to have been previously studied under trial conditions in a head-tohead comparison with lithium, meaning at least comparable short-term effectiveness between the two treatments under trial conditions is known [20]. While this study by Bauer and colleagues reported quetiapine to be noninferior to lithium over a six week treatment course, there was also some indication that quetiapine may be more clinically effective than lithium. This included lower depression severity scores (Montgomery-Åsberg Depression Rating Scale, MÅDRS), reported as early as day 4 of treatment, and less sleep disturbance [20]. Additionally, a network meta-analysis comparing augmentation agents in TRD found lithium and quetiapine to both be more effective than a placebo, but indicated that the efficacy of quetiapine was more robust than lithium [27].

\section{Objectives and hypothesis}

This study aims to determine whether the decision to prescribe quetiapine is more clinically and cost effective than the decision to prescribe lithium over a longer term 
in a randomised clinical trial powered to detect superiority. We propose a 12 month follow up period, hypothesising that quetiapine will be superior to lithium in terms of our primary outcomes: time to all-cause treatment discontinuation and average depressive symptom burden over 12 months.

We hope to help shape a modified treatment pathway for TRD in which one augmenter is preferential to the other. However, if quetiapine is not found to be clinically superior based on our primary and secondary outcomes, the results of the cost effectiveness analysis, or individual level predictive factors, may determine the most appropriate choice for each patient.

The trial will recruit patients with TRD: defined as the failure to adequately respond to two or more therapeutic trials of antidepressant treatments in the current episode (at adequate dose and duration) [3, 8]. This definition of treatment resistance is in line with the point at which treatment augmentation is recommended in clinical practice [3] and at which the evidence suggests clinical equipoise between the two treatments [20].

This protocol paper is consistent with SPIRIT (Standard Protocol Items: Recommendations for Interventional Trials) 2013 recommendations [28] (checklist attached).

\section{Methods/design}

\section{Study design}

LQD is a phase 4, 12-month, parallel group, multi-centre, patient 1:1 randomised, pragmatic, open-label, superiority trial, comparing the clinical and cost-effectiveness of the decision to prescribe lithium versus quetiapine as add-on treatment to antidepressant medication in patients with TRD.

\section{Participants}

We will recruit participants who fulfil the following inclusion criteria:

1. Under the care of a GP and/or adult mental health services

2. Current episode of depression meeting Diagnostic and Statistical Manual of Mental Health Disorders - Fifth Edition (DSM-5) [29] criteria for MDD (single or recurrent episode) assessed using the Mini International Neuropsychiatric Interview, Version 7 (MINI 7.0) [30]

3. HDRS - 17 item [31] score $\geq 14$ at screening

4. Any gender and aged 18 years or over

5. TRD $[3,32]$ : defined as failing to adequately respond to at least two antidepressant therapies, prescribed for at least 6 weeks at minimum therapeutic dose, as determined by the Maudsley Prescribing Guidelines (MPG) and/or British National Formulary (BNF) $[33,34]$
6. Current antidepressant treatment has remained unchanged for $\geq 6$ weeks

7. Provision of written, informed consent

Exclusion criteria for participants are:

1. Diagnosis of bipolar disorder (defined as meeting DSM-5 criteria for bipolar 1 or 2) on the MINI 7.0

2. Diagnosis of current psychosis

3. Use of lithium or quetiapine during current depressive episode

4. Ongoing use of another atypical antipsychotic

5. Known contraindication to use of either lithium or quetiapine

6. Currently participating in another clinical trial of an investigational medical product

7. Insufficient degree of comprehension or attention to be able to engage in trial procedures

8. Pregnancy, actively trying for pregnancy, or breastfeeding

\section{Recruitment procedure and assessments}

Participant recruitment will take place across three of the UK National Institute of Health Research (NIHR) Clinical Research Network hubs: South London, the North East and North Cumbria, and Thames Valley and South Midlands. Recruitment is underway at four sites within these hubs: the South London and Maudsley National Health Service (NHS) Foundation Trust (SLaM); Northumberland, Tyne and Wear NHS Foundation Trust (NTW); Oxford Health NHS Foundation Trust (OHT); and Tees, Esk and Wear Valleys NHS Foundation Trust (TEWV).

Potential participants will be identified primarily at routine secondary care clinic appointments or via Consent for Contact initiatives within the trusts (e.g. http:// www.slam.nhs.uk/research/patient-involvement/current-opportunities/consent-for-contact) alongside advertisements, and via Participant Identification Centres including primary care and external UK NHS trusts to enhance recruitment.

\section{Randomisation and blinding}

The study will randomise individual participants 1:1 to the decision to prescribe lithium or quetiapine, stratified by recruiting region (London, Oxfordshire, or North East England), depression severity (HDRS-17), and TRD severity (failure of two / three or more antidepressant treatments in the current episode), using block randomisation with randomly varying block size. Randomisation will be conducted by a trial researcher using an independent online service provided by UK Clinical Research Collaboration registered King's Clinical Trial Unit, with unique patient identification numbers generated by the InferMed MACRO system.

This is an unblinded study, whereby treating clinicians, trial researchers, and patients will be aware of the treatment 
allocation for patients where applicable to their role. In order to reduce the potential for assessor bias, the following clinician-rated assessments will be completed at follow up visits by an assessor who is blind to the patient's medication (face-to-face or via phone): 1) MÅDRS [35], and 2) Clinical Global Impressions scale (CGI) [36]. Trained trial researchers and clinicians may perform blinded assessments, but must not have access to the trial database for the relevant site to reduce the likelihood of unblinding (access to the trial database is granted on a site by site basis, dependent on role). The blinded assessor will remind the participant not to reveal treatment allocation at the start of the assessment and will confirm they remained blinded to treatment allocation throughout the assessment.

\section{Primary outcomes}

a) Difference in time to all-cause treatment discontinuation over 12 months post-randomisation between lithium and quetiapine using survival analysis methods, with time being between the first prescription and discontinuation of the medication to which the patient was randomised.

b) Longitudinal Depression Severity: the difference in the area under the curve between lithium and quetiapine over 12 months post-randomisation in the self-rated Quick-Inventory of Depressive Symptomatology (QIDS-SR) [37], assessed weekly via the True Colours system (www.truecolours.nhs.uk), using a linear mixed model, covarying for baseline QIDS-SR score.

\section{Secondary outcomes}

The secondary outcomes will be measured at 8 and 52 weeks, and differences between lithium and quetiapine assessed at both time points. Continuous secondary outcomes measured over time will use appropriate generalised linear models, covarying by baseline score where applicable. Where there are repeated measures of a continuous variable, the analysis will be set in the mixed model framework. Time to event variables will be analysed using survival methods. The secondary outcomes are as follows:

- Clinician-rated depression severity (continuous total score on the MÅDRS).

- Clinician-rated response and remission rates (defined as proportion with $\geq 50 \%$ reduction in baseline MÅDRS total score and MÅDRS total score $\leq 10$, respectively).

- Global improvement (proportion of patients with a CGI-improvement score of 'much' or 'very much improved').

- Health-related quality of life (summary index EuroQol-5D, EQ-5D score [38]).
- Social functioning (continuous total Work \& Social Adjustment Scale (WSAS) score [39]).

- Self-report adherence to treatment (5-item Medication Adherence Report (MARS-5) continuous total scores and exploratory cut-offs categorising patients as either adherent or nonadherent [40]).

- Physical health parameters: weight $(\mathrm{kg})$ and blood pressure (mmHg).

- Time to commencement of trial medication from randomisation (and number of patients commencing therapy in each arm).

- Time to uptake of a new intervention for depression from randomisation (pharmacological or nonpharmacological) over 12 months (and proportion of patients in each arm receiving new interventions).

- Side Effects (continuous total score on the Patient Rated Inventory of Side Effects (PRISE) [41]).

- Frequency of Serious Adverse Events (SAEs).

Secondary outcomes, except for SAEs, will be adjusted for multiple comparisons. SAEs will be tabulated as number of SAEs in each group, and number of people reporting an SAE in each group over 12 months. We will also summarise the reasons for clinicians not prescribing and patients not commencing the medication they are randomised to, reasons for treatment discontinuation, the number of withdrawals, and the reasons for withdrawals from the trial. However, we will not test the difference between the groups on these variables statistically.

\section{Additional outcomes}

For a full list of tertiary and ancillary trial outcomes please visit clinicaltrials.gov. Two key ancillary outcomes are detailed below:

- Cost-effectiveness: This data will be gathered from the Client Service Receipt Inventory (CSRI) [42], modified for TRD, asked at baseline, 8, 26, and 52 week study visits. Cost-effectiveness analyses will be conducted from a health and social care, and a societal perspective. This will include trial and concomitant medication costs, healthcare costs (including hospital, community-based, social and primary care services), costs to statutory and non-statutory services, impact on caregivers and families, and days off work due to health problems.

- Predictors of treatment response: for example, baseline staging of treatment resistance (Maudsley Staging Method, MSM [32]), depression characteristics (e.g. severity (HDRS [31]), chronicity, family history, recurrence (MINI 7.0), psychiatric comorbidity (MINI 7.0), subtype (e.g. typical versus atypical, clinician rated Inventory of Depressive 
Symptomatology (IDS-C) [43]), personality (Standard Assessment of Personality [44]), type of antidepressant (e.g. selective serotonin reuptake inhibitor (SSRI) vs. non-SSRI), hypomanic symptoms (16-item hypomanic checklist (HCL-16) [45] at baseline) and sociodemographic factors (e.g. sex, age, ethnicity, body mass index). Briefly, the analysis would build a predictive model of response using an appropriate variable selection procedure (i.e. manual forward stepwise regression).

\section{Interventions}

As this is a pragmatic study, trial medications will be prescribed open-label and no measures will be taken to intervene with patients' medication adherence. Trial clinicians will be advised to keep patients' existing antidepressant treatment(s) at a stable dose within the therapeutic range, as defined in the MPG and BNF [33,34]. The following recommendations for titration and dosing of each of the two treatment arms will be provided, in line with current best practice guidelines [33, 34]. However failure to adhere to these guidelines will not constitute a protocol deviation:

Lithium arm: lithium carbonate/citrate, added on to the current antidepressant. The dose should be adjusted to achieve a serum-lithium concentration of $0.4-1.0 \mathrm{mmol} / \mathrm{l}$ $12 \mathrm{~h}$ after a dose. Serum level checks should be performed on days 4-7 of treatment and be repeated every week until dosage has remained constant for 4 weeks aiming for an optimal therapeutic plasma level of $0.6-1.0 \mathrm{mmol} / \mathrm{l}[22,33]$. Quetiapine arm: quetiapine fumarate (XR or immediate release formulation) added on to the current antidepressant, taken once daily before bedtime. Recommended dose titration: $50 \mathrm{mg}$ on days 1 and 2 and $150 \mathrm{mg}$ on day 3 , aiming for a dose of $300 \mathrm{mg} /$ day by week 2 , if tolerated. Thereafter, flexible dosing will follow in the range 150-300 mg/day according to tolerance (as per Bauer et al. 2013) [23]. In elderly patients ( $>65$ years old), the dose titration protocol should be: $50 \mathrm{mg} /$ day on days $1-3$, increasing to $100 \mathrm{mg} /$ day on day $4,150 \mathrm{mg} /$ day on day 8 and $300 \mathrm{mg} /$ day not before day 22 of treatment, if required.

Dosing regimens may need to be altered in the case of concomitant administration of drugs that interact with either quetiapine or lithium.

The initial prescription of a study medication and any essential pre-prescription safety checks must be overseen by a trial clinician. This is followed by a fully flexible continuation phase for up to 52 weeks post-randomisation, in which treatment is shared between primary and secondary care as appropriate according to standard NHS practice in the relevant trust.
In this trial, patients will be randomised to the decision to prescribe either lithium or quetiapine. This means that in each case, the treating trial clinician will decide whether or not to prescribe the medication that the patient has been randomised to, according to their clinical judgement. It is therefore possible that a small proportion of randomised patients will not receive the medication that they are allocated to. This may be due to a previously unknown contraindication to the study medication arising during the pre-prescribing safety checks (exclusion criteria for trial entry is known contraindications to lithium or quetiapine only). The required safety checks are: electrocardiogram, if clinically indicated (both treatment arms) and pre-lithium blood tests (renal function, thyroid function, full blood count, serum calcium). These tests must be conducted prior to prescription, unless they have been completed within a sufficiently recent period according to the prescribing clinician's judgement.

All patients will be followed up for 12 months, regardless of medication status. It is expected that a significant proportion of patients will fail to respond or tolerate the medication and will terminate treatment before 12 months. Treatment may also be extended beyond the follow up period at the discretion of the patient's clinician. All concomitant pharmacological and non-pharmacological interventions are permitted throughout the duration of the trial and will be recorded by the trial team.

\section{Assessments}

All assessments, data entry and randomisation will be conducted by trained trial researchers with oversight from the clinical chief (AJC) and principal investigators (AHY, RHMW, JG, RN). See Table 1 for details of all study measures and Fig. 1 for a flow chart of trial procedures.

\section{Screening visit}

All participants must provide written informed consent at the start of the screening assessment. Consent may be taken by a trial clinician, or delegated non-clinical researcher with trust approval. Confirmation of eligibility will be signed off by a trial clinician at the end of this assessment.

\section{Baseline, weekly monitoring and follow up visits}

If the baseline assessment is more than 7 days after the screening visit, the screening assessments (excluding demographic information) will be repeated to ensure that the participant still meets the required eligibility criteria. Randomisation will be conducted at Week/Time Point 0 , which is the day of the baseline visit. Follow-up assessments with trial researchers will be conducted at $8(+-1)$ weeks, 26 $(+-2)$ weeks, and $52(+-2)$ weeks post-randomisation.

Patients will also be asked to complete three questionnaires weekly via the True Colours system (https://truecolours.nhs.uk): the QIDS-SR, WSAS, and study specific 
Table 1 Summary of measures

\begin{tabular}{|c|c|c|c|c|c|c|}
\hline \multirow[t]{2}{*}{ Time point } & \multirow[t]{2}{*}{ Screening } & \multirow{2}{*}{$\begin{array}{l}\text { Baseline } \\
\text { (Week 0) }\end{array}$} & \multicolumn{4}{|l|}{ Follow up } \\
\hline & & & $\begin{array}{l}\text { Weekly } \\
\text { Assessments } \\
\text { Weeks 0-52 }\end{array}$ & $\begin{array}{l}\text { Week } 8 \\
(+-1 \text { weeks) }\end{array}$ & $\begin{array}{l}\text { Week } 26 \\
(+-2 \text { weeks })\end{array}$ & $\begin{array}{l}\text { Week } 52 \\
(+-2 \text { weeks })\end{array}$ \\
\hline Written informed consent & $\sqrt{ }$ & & & & & \\
\hline Assessment of eligibility & $\sqrt{ }$ & & & & & \\
\hline MINI 7.0 to confirm MDD and other comorbid axis 1 disorders & $\sqrt{ }$ & & & & & \\
\hline Assessment of depression severity (HDRS-17) & $\sqrt{ }$ & & & & & \\
\hline Assessment of medication history in current depressive episode & $\sqrt{ }$ & & & & & \\
\hline $\begin{array}{l}\text { Sociodemographic / Psychiatric and Medical History } \\
\text { (including MSM) }\end{array}$ & $\sqrt{ }$ & & & & & \\
\hline $\begin{array}{l}\text { Assessment of concomitant medication and } \\
\text { non-pharmacological therapies }\end{array}$ & $\sqrt{ }$ & $\sqrt{ }$ & & $\sqrt{ }$ & $\sqrt{ }$ & $\sqrt{ }$ \\
\hline Clinician-rated assessment of clinical symptoms (MÅDRS, CGI) & & $\sqrt{ }$ & & $\sqrt{ }$ & $\sqrt{ }$ & $\sqrt{ }$ \\
\hline Randomisation & & $\sqrt{ }$ & & & & \\
\hline Clinician-rated depression severity (including subtype: IDS-C) ${ }^{b}$ & & $\sqrt{ }$ & & $\sqrt{ }$ & & \\
\hline Hypomanic checklist (HCL-16) ${ }^{\text {b }}$ & & $\sqrt{ }$ & & & & \\
\hline Assessment of side effects (FIBSER ${ }^{\mathrm{b}}$ and PRISE) & & & & $\sqrt{ }$ & $\sqrt{ }$ & $\sqrt{ }$ \\
\hline Assessment of quality of life (EQ-5D) & & $\sqrt{ }$ & & $\sqrt{ }$ & $\sqrt{ }$ & $\sqrt{ }$ \\
\hline Assessment of cognition (THINC-it ${ }^{a, b}$ and DSCT ${ }^{b}$ ) & & $\sqrt{ }$ & & $\sqrt{ }$ & $\sqrt{ }$ & $\sqrt{ }$ \\
\hline $\begin{array}{l}\text { Weekly True Colours self-rated measures: QID-SR, WSAS, and trial } \\
\text { medication status }\end{array}$ & & $\sqrt{ }$ & $\sqrt{ }$ & $\sqrt{ }$ & $\sqrt{ }$ & $\sqrt{ }$ \\
\hline $\begin{array}{l}\text { Self-Rated clinical measures (ASRM }{ }^{b} \text {, Maudsley VAS measures }{ }^{b} \text {, } \\
\text { GAD- } 7^{b}, \text { SAPAS }^{b} \text { ) }\end{array}$ & & $\sqrt{ }$ & & $\sqrt{ }$ & $\sqrt{ }$ & $\sqrt{ }$ \\
\hline Assessment of costs (CSRI ${ }^{\mathrm{b}}$ and employment status $\left.{ }^{\mathrm{b}}\right)$ & & $\sqrt{ }$ & & $\sqrt{ }$ & $\sqrt{ }$ & $\sqrt{ }$ \\
\hline Treatment satisfaction $\left(\mathrm{TSQM}^{\mathrm{b}}\right)$ & & & & $\sqrt{ }$ & $\sqrt{ }$ & $\sqrt{ }$ \\
\hline $\begin{array}{l}\text { Adherence (baseline to antidepressant }{ }^{\mathrm{b}} \text {, follow up } \\
\text { to trial medication) }\end{array}$ & & $\sqrt{ }$ & & $\sqrt{ }$ & $\sqrt{ }$ & $\sqrt{ }$ \\
\hline Qualitative assessment of patient experience of True Colours ${ }^{a, b}$ & & & & $\sqrt{ }$ & or $\sqrt{ }$ & or $\sqrt{ }$ \\
\hline $\begin{array}{l}\text { Physical health (weight, height, blood pressure, } \\
\text { pulse rate, waist circumference) }\end{array}$ & & $\sqrt{ }$ & & $\sqrt{ }$ & $\sqrt{ }$ & $\sqrt{ }$ \\
\hline Blood tests (FBC, U\&Es, LFTs, TFT, glucose, lipids, calcium) & & $\sqrt{ }$ & & $\sqrt{ }$ & & $\sqrt{ }$ \\
\hline Lithium and quetiapine serum levels $s^{a, b}$ & & & & $\sqrt{ }$ & & $\sqrt{ }$ \\
\hline BioResource genetic/cortisol/cytokine sample collection ${ }^{a, b}$ & & $\sqrt{ }$ & & $\sqrt{ }$ & & $\sqrt{ }$ \\
\hline
\end{tabular}

MINI 7.0 Mini International Neuropsychiatric Interview, Version 7.0, MDD major depressive disorder, HDRS Hamilton Depression Rating Scale - 17 items, MSM Maudsley Staging Method, MÅDRS Montgomery-Åsberg Depression Rating Scale, CGI Clinical Global Impressions, IDS-C Inventory of Depressive Symptomatology Clinician Rated, HCL-16 Hypomanic Checklist - 16 items, FIBSER Frequency, Intensity and Burden of Side Effects Ratings, PRISE Patient Rated Inventory of Side Effects, EQ-5D EuroQol-5D health index, THINC-it THINC-it tool for cognitive dysfunction in Major Depressive Disorder, DSCT Digit Symbol Coding Test, WSAS Work and Social Adjustment Scale, ASRM Altman Self-Rating Mania Scale, VAS Visual Analogue Scale, GAD-7 Generalised Anxiety Disorder questionnaire - 7 items, SAPAS Standard Assessment of Personality: abbreviated Scale, CSRI Client Service Receipt Inventory, FBC Full blood count, U\&Es Urea, electrolytes and creatinine, LFTS Liver function tests, TFT Thyroid function tests

a Optional and/or collected in a subset of participants

${ }^{\mathrm{b}}$ Measures solely for tertiary and ancillary analyses

questions about medication status. True Colours is an online monitoring tool on which questionnaires can be completed via email, text, or by logging on to the website. For patients without internet access, a paper version can be provided. Automated reminders will be sent to participants on a day and time of their choosing.

Optional study blood tests will be conducted at baseline, 8 and 52 week visits (agreement to clinical blood tests throughout the trial duration is not optional). Optional blood tests include samples for genetic and cytokine analysis, along with hair samples to assess cortisol levels, and saliva samples for genetic analysis where blood samples are not provided. This will be done in collaboration with the Biomedical Research Council (BRC) BioResource. Further optional measures include a qualitative interview exploring patient experience of True Colours as a mood monitoring tool (conducted at one follow up appointment) and the THINC-it tool for cognitive function [46]. 


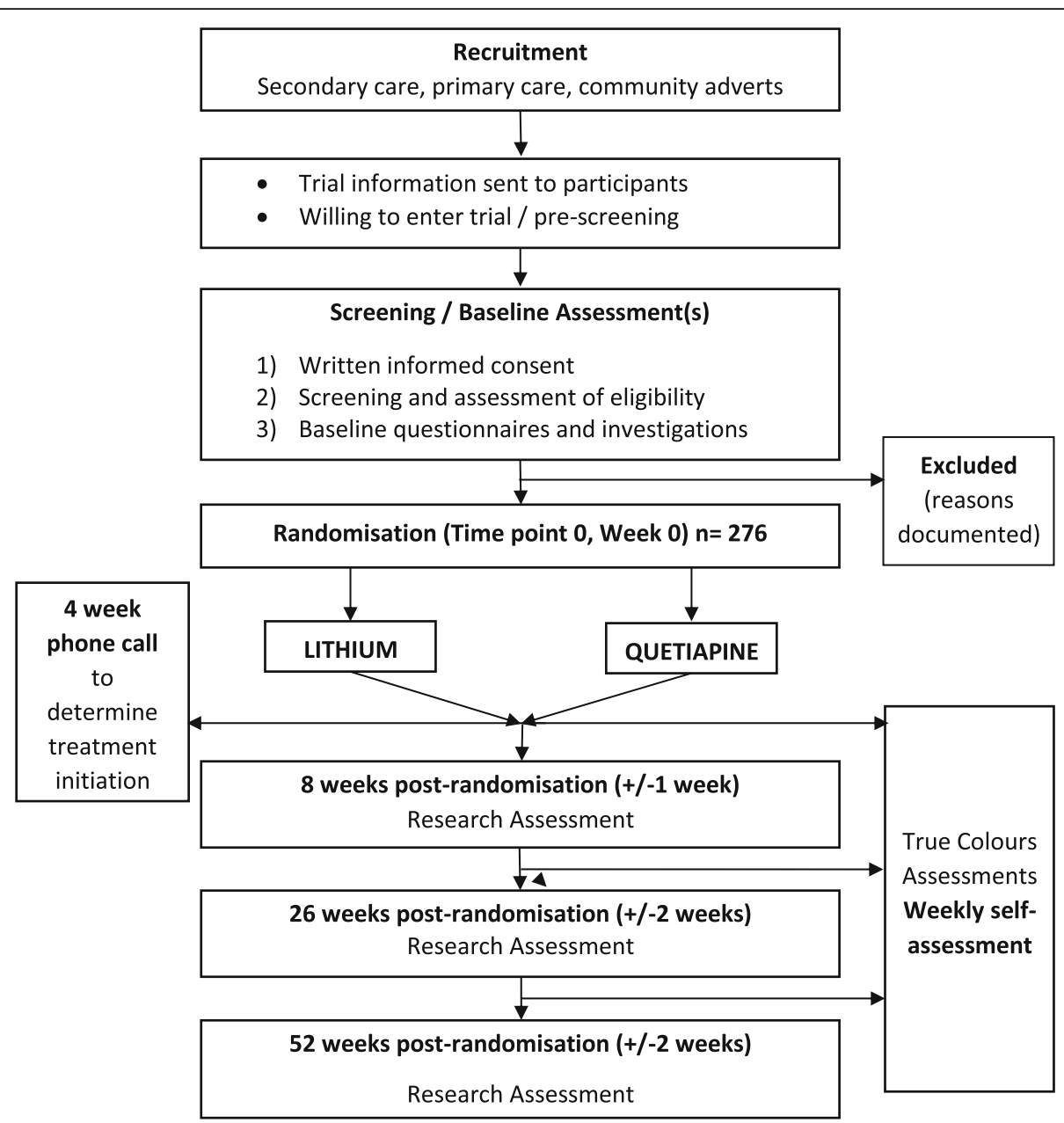

Fig. 1 Flow Chart of Trial Procedures

One section of the CSRI collects data about the patient's principal formal or informal carer. Where applicable, the carer can complete this questionnaire following their provision of written informed consent. Where this is not possible, responses from the patient will be accepted.

At 4 weeks post-randomisation, participants will receive a phone call from a trial researcher to determine the date they began taking their allocated medication. If applicable, the reason the patient has not commenced their medication will be recorded and this question will be repeated at follow-up visits to determine if a patient has subsequently begun the allocated medication.

All study data will be stored on a custom-designed, online data entry system which is compliant with Good Clinical Practice guidelines, and created and maintained by King's Clinical Trials Unit (InferMed MACRO version 4.0). The system is programmed to perform validation checks for data quality purposes (e.g. range checks to avoid data entry errors) and flags out of range or missing data for monitoring purposes. It also has a built in audit trail to aid data monitoring.
Statistical methods for analysing primary and secondary outcomes

A full statistical analysis plan will be drawn up prior to completion of data collection. The main analysis will follow an intention to treat (ITT) principle, whereby patient data are analysed by treatment group, regardless of the medication status of patients throughout the follow-up period.

Participant flow will be presented in a CONSORT diagram. For analysis of the listed primary and secondary outcomes, missing baseline data will be filled in using imputation [47]. Models will covary for randomisation stratification factors throughout. For the self-report QIDS-SR score a linear mixed model, covarying by baseline score, will be used for the estimation of the mean treatment difference and 95\% confidence interval (CI) over the course of the trial. Full information maximum likelihood methods will be used for analysis, which will account for missing data under the missing at random assumption. Error distributions will be checked using Q-Q plots. Time to discontinuation will be calculated from first prescription date to treatment discontinuation, and will be analysed 
using Kaplan-Meier methods and Cox regression models, providing the hazard ratio for treatment discontinuation in lithium versus quetiapine, and associated 95\% CI.

Continuous secondary outcomes measured over time will use generalised linear models appropriate to the variable distribution covarying by baseline where applicable, and using mixed models where there are repeated measurements. Adjustment for multiple testing will be presented [48].

For measures where missingness is above $5 \%$ and where the absence of repeated measurement gives less confidence in the adequacy of the missing data properties of maximum likelihood, we may undertake a sensitivity analysis within a multiple imputation framework [49].

\section{Power calculation}

Based on our power calculation and superiority design, we require 276 patients to be randomised. When considering the sample size required, we have considered both the primary outcome measures of time to all cause treatment discontinuation and longitudinal symptom severity as assessed by QIDS-SR scores. This sample size was calculated based on the following assumptions:

- Treatment discontinuation: estimated $20 \%$ discontinuation from treatments by 8 week follow up visit (as per Bauer et al., 2013) [20]), and 50\% treatment discontinuation for lithium and 30\% for quetiapine by 52 weeks.

- Missing data: $40 \%$ missingness for each of the postrandomisation QIDS-SR scores was allowed for. While the missingness has been assumed independent over time, the QIDS-SR scores are assumed uniformly correlated with a correlation of 0.6 [47]. We have allowed the discontinuation status at follow-up to be unknown for $10 \%$ of the sample.

- Effect size: The minimum clinically significant difference for outcomes in depression treatment is widely taken to be 3 points on the HDRS $[3,50]$. This corresponds to an effect size of 0.38 between treatments. We wish to see a difference of this effect size in the QIDS-SR score sustained over the duration of the trial and so will estimate the effect as an area under the curve.

- Power: All power calculations are for two-tailed tests and alpha $=0.05$.

With a sample size of 276 at baseline and 10\% loss to follow up, we expect 248.4 patients to complete the trial. The Stata stpower command gave $90 \%$ power using a logrank test to detect a difference in the time to discontinuation with 50\% (lithium) and 70\% (quetiapine) as the proportions of participants remaining on assigned treatment. This determined the lower limit of the sample size. Applying this sample size to the self-report QIDS-SR data we also needed to account for the likely haphazard nature of missing data points at each assessment time point. We used simulation and the non-central chi-square method. Using 1000 samples, a simple random intercept model covarying for baseline, a time dummy variable and with a single average combined treatment effect, this number of participants will provide $99.7 \%$ power to detect an effect size of 0.38 (with 1 degree of freedom, $X^{2}=3.84$, and non-centrality parameter $=20.86$ ).

\section{Monitoring}

There is no planned interim analysis and no specific "stopping rules" for the study. We have an independent Data Monitoring and Ethics Committee and an independent Trial Steering Committee. SAEs will be reported to the relevant bodies for all patients throughout their involvement in the trial. Clinicians have the right to discontinue the study drug in the event of identified contraindications, inter-current illness, pregnancy, adverse drug reactions, adverse events, protocol violations, administrative causes, or for other reasons. We will adhere to NHS confidentiality practice, and to the Research Governance Framework in monitoring, auditing and managing the research.

\section{Discussion}

This protocol paper describes a pragmatic randomised clinical trial designed to determine whether the decision to prescribe quetiapine is more clinically and cost effective than lithium for patients with TRD over 12 months. The chronicity of TRD, in which patients often fluctuate between states of remission and relapse [51], highlights not only the importance of this research question, but also the implementation of a 12 month follow up period. As discussed, there have been no direct head-to-head comparisons of these treatments with a follow up period of greater than six weeks. This is insufficient given that up to $80 \%$ of patients with TRD relapse within 12 months of responding to treatment $[8,20]$. Our choice of primary outcome, a longitudinal measure of depression severity (QIDS-SR), measured weekly over 12 months via the True Colours system (www.truecolours.nhs.uk), recognises the highly fluctuating nature of this disorder. True Colours has been used to assess mood in prior research studies with success [52] and facilitates the monitoring of all cause treatment discontinuation across the full duration of the trial.

The benefits of determining whether one of these recommended and widely prescribed medications is more effective for patients with TRD are clear. However the results of this study should also positively impact secondary care mental health teams and the wider NHS by helping to minimise relapse rates and in doing so alleviate some of the financial and time burden placed 
on mental health services. If there is no significant difference between lithium and quetiapine in the primary outcomes of clinical effectiveness, the cost-effectiveness analysis, which takes into account the different monitoring requirements for these medications, may benefit health bodies by informing them of the most costeffective of these two treatment options. In addition, our further analyses may contribute to the growing body of research examining predictors of treatment response in depression, and the development of personalised mental health care.

The potential impact of this trial is maximised by its pragmatic design, which ensures that the results will be directly applicable to real-world clinical practice. The PRECIS-2 tool [51] was used to assess how far we have achieved this and what elements of the design are most pragmatic. A key pragmatic strength is our choice of primary outcomes (the effectiveness and tolerability of the treatments) which are directly applicable to, and can clearly be understood by, the patient group. Additionally, our broad inclusion criteria and minimal exclusion criteria enable us to capture as much variation in the TRD population as possible and include the majority of patients that are likely to receive such an augmentation therapy in routine clinical practice. Examples of this are the inclusion of patients demonstrating suicidal ideation, as this group is often excluded from clinical trials, and the decision not to include an upper age limit (commonly set at 65 years for TRD clinical research) improving the applicability of our results to older patients [3]. Furthermore, alongside the recruitment of patients from secondary care settings, where lithium or atypical antipsychotic augmentation is commonly initiated, we are also recruiting patients from primary care. In doing so we are ensuring that our samples reflect the range of patients that should be considered for these two augmentation therapies according to best practice guidelines.

The pragmatic design of the trial is also reflected in our decision to not dictate a specific dosing or monitoring schedule. As discussed, clinicians are provided with bestpractice dosing and monitoring guidelines, but are free to prescribe, monitor and discontinue the trial medication as they find clinically appropriate.

The PRECIS-2 tool also highlighted some necessary limitations to the trial's pragmatism; primarily the large number of measures conducted during the follow up period. As with all pragmatically designed trials, there must be a balance between the need for adequate data collection and the additional burden this places on participants, beyond what would be experienced in normal clinical practice. However, all chosen measures were deemed necessary by the study team to ensure that the results of the trial aid the development of a modified and evidence based treatment pathway for TRD, and contribute to the improvement of treatment outcomes in this patient group. Conducting a thorough psychiatric history assessment to determine TRD staging and depression characteristics, assessing personality traits relating to refractoriness, evaluating mixed and atypical depressive symptomatology, and collecting biological samples to examine genetic and inflammatory markers and changes in physical health, will allow for a thorough comparison between the two treatments. In order to minimise patient dropout, some of the measures associated with tertiary or ancillary outcomes only have been made optional for participants. Additionally, information will be gathered from medical records or at future follow up visits where possible should a patient fail to attend or complete an assessment.

The LQD study is a large scale trial designed to build on evidence reported by previous short term comparisons of lithium and quetiapine augmentation in patients with TRD $[21,22]$. In doing so, we hope to definitively determine which of these two augmentation treatments (if either) should be preferential over the other, improving patient outcomes and minimising the burden that TRD places on clinical resources.

\section{Trial status}

Recruitment is currently ongoing at 4 sites (SLaM, NTW, $\mathrm{OHT}$, and TEWV) and is set to continue until February 2019. It is anticipated that the trial will end in June 2020.

\section{Abbreviations}

ASRM: Altman Self-Rating Mania Scale; BAP: British Association for Psychopharmacology; BNF: British National Formulary; CGI: Clinical Global Impressions; Cl: Confidence interval; CSRI: Client Service Receipt Inventory; DSCT: Digit Symbol Coding Test; DSM-V: Diagnostic and Statistical Manual of Mental Disorders - Fifth Edition; ECG: Electrocardiogram; EQ-5D: EuroQol-5D health index; FBC: Full blood count; FIBSER: Frequency, Intensity and Burden of Side Effects Ratings; GAD-7: Generalised Anxiety Disorder questionnaire 7 items; HCL-16: Hypomanic Checklist - 16 items; HDRS-17: Hamilton Depression Rating Scale - 17 items; IDS-C: Inventory of Depressive Symptomatology - Clinician Rated; ITT: Intention to treat; LFTS: Liver function tests; LQD: Lithium versus Quetiapine in Depression; MÅDRS: Montgomery-

Åsberg Depression Rating Scale; MARS-5: Medication Adherence Report Scale - 5 items; MDD: Major depressive disorder; MINI 7.0: Mini International

Neuropsychiatric Interview, Version 7.0 for DSM-5; MPG: Maudsley Prescribing Guidelines; MSM: Maudsley Staging Method; NHS: National Health Service; NICE: National Institute for Health and Care Excellence; NIHR: National Institute of Health Research; NTW: Northumberland, Tyne and Wear NHS Foundation Trust; OHT: Oxford Health NHS Foundation Trust; PRISE: Patient Rated Inventory of Side Effects; QIDS-SR: Quick Inventory of Depressive Symptomatology - Self-Rated; quetiapine XR: quetiapine extended release; SAEs: Serious Adverse Events; SAPAS: Standard Assessment of Personality: abbreviated Scale; SLaM: South London and Maudsley NHS Foundation Trust; SPIRIT: Standard Protocol Items: Recommendations for Interventional Trials; SSRI: Selective serotonin reuptake inhibitor; TEWV: Tees, Esk and Wear Valleys NHS Foundation Trust; TFT: Thyroid function tests; THINC-it: THINC-it tool for cognitive dysfunction in major depressive disorder; TRD: Treatment resistant depression; TSQM: Treatment Satisfaction Questionnaire for Medication; U\&Es: Urea, electrolytes and creatinine; VAS: Visual Analogue Scale; WSAS: Work and Social Adjustment Scale 


\section{Acknowledgements}

The trial is co-sponsored by King's College London, and the South London and Maudsley NHS Foundation Trust. LQD has an independent Trial Steering Committee (TSC; members: David Baldwin (chair), Jasmine Stuart Smith, Joanna Clarke, Mohamed Abdelghani, and Peter Haddad) and independent Data Monitoring and Ethics Committee (DMEC; members: Mike Bradburn (chair), Christopher Dickens, and Louise Marston). For details of the TSC and DMEC charters and roles, please contact the corresponding author. We thank the contribution of Patient and Public Involvement (PPI) members who provided feedback and valuable suggestions in the development of this trial and the study documents. Following PPI consultation, we increased the frequency of self-report depression ratings on True Colours (weekly, rather than less frequently) due to patient experience that this would give a more accurate indication of long term symptom control and additionally be beneficial to patients. We also included a 26 week face-to-face study visit (the original proposal was follow up visits at 8 and 52 weeks only). The authors would like to thank those who have helped in setting up the study: Caroline Loveland, Lauren Moult, Jennifer Liebscher, Dunstan Nicol-Wilson, Ilia Bountouni, Mario Juruena, Emma Incecik, Lyndsey Dixon, Stuart Watson, Vanashree Wadekar, Bradley Mullins, Gordon Riddell, Simon Douglas, Alvaro Barrera, Dominic Wilson, Carol Bell, Catherine Smith, Kate Byrnes, Elka Giemza, Andrew Molodynski, Hielkje Verbrugge, Janet Wingrove, Aleksandra Litovski, Megan Pritchard, Susanne Fischer, Rebecca Strawbridge, Bartek Pliszka. We would also like to thank members of the UK NIHR Clinical Research Networks involved in the trial for their help with recruitment and site set up: South London CRN (Rani Badhan, Andra Cosma, Praveen Macherla, Simon Davies, Ana Guerra, Catarina Guerreiro, Amy McLachlan), Thames Valley and South Midlands CRN (Alexandra Forrest, Jennifer Potts, Elizabeth llett, Ros Humphreys), and the North East and North Cumbria CRN (Wendy Hall, Susan Bonner, Aileen Henderson). We gratefully thank the South London and the Maudsley BioResource staff including the NIHR Biomedical Research Centre at SLaM and King's College London for funding for this collaboration. Further information can be found at http://brc.slam.nhs.uk/about/core-facilities/bioresource. Special thanks to Cerisse Gunasinghe, Alvin Romero, Kate Fayer, Adewale Odesanya, and Gerome Breen in the team.

Thanks to King's Clinical Trials Unit for providing us with our randomisation and database services. In particular Caroline Murphy, Jade Higman, Aleksandra Kata, Joanna Kelly and Evangelos Georgiou for their help in designing and programming these services. We also thank members of King's Health Partners Clinical Trials Office including Katarzyna Smigielska, Emma Tylor, Helen Critchley and Ingrid Brumarescu.

\section{Funding}

The project is funded with a $£ 1,075,315$ grant by the NIHR Health Technology Assessment (HTA) programme (reference: 14/222/02). We additionally gratefully acknowledge capital equipment funding from the Maudsley Charity (grant reference: 980) and Guy's and St Thomas's Charity (grant reference: STR130505) for biological sample collection. Additionally, this report represents independent research funded by the NIHR/Wellcome Trust King's Clinical Research Facility and NIHR BRC at South London and Maudsley NHS Foundation Trust, Northumberland, Tyne and Wear NHS Foundation Trust, and Oxford Health NHS Foundation Trust and King's College London, the University of Oxford, and Newcastle University. The views expressed are those of the authors and not necessarily those of the NHS, the NIHR, or the Department of Health.

The funders and sponsors have not, and will not, participate in the study design; data collection; management; analysis, and interpretation of data, or writing of reports: they do not have the authority over the decision to submit reports for publication or the conclusions herein.

\section{Availability of data and materials}

Not applicable.

\section{Authors' contributions}

AJC, LM, AHY, RHMW, JG, AP, RT, DD, AC, MJA, RN, KG, RH, RR and JH were involved in the conception of the trial, study design and were key to trial set up and recruitment. Funding was obtained by AJC, AHY, RHMW, JG, AP, RR, AC, \& DD. Statistical and trial design guidance was provided by $K G, R H, R R$ and $A P$. $L M, R T$ and AJC were responsible for drafting the manuscript with substantial input from all other authors. All authors reviewed, revised and approved the final manuscript.

\section{Ethics approval and consent to participate}

The study was approved by: the Cambridge-South Research Ethics Committee (REC), United Kingdom (registration number: 16/EE/0318); the Medicines and Healthcare products Regulatory Authority (MHRA: EudraCT: 2016-001637-27); and the Health Research Authority (HRA). All participants will give written informed consent before engaging in any trial related activities. Recruitment began in December, 2016. The currently approved protocol is version 3.0, dated 20.12.2016. Amendments will be sent for approval to the REC, HRA and MHRA as appropriate and protocol amendments addressed at ISRCTN (http:// www.isrctn.com), clinicaltrials.gov and communicated to the study team and participants as required.

\section{Consent for publication}

Not applicable.

\section{Competing interests}

AJC has in the last three years received honoraria for speaking from Astra Zeneca (AZ) and Lundbeck, honoraria for consulting from Allergan and Livanova and research grant support from Lundbeck. AHY has given paid lectures and sits on advisory boards for all major pharmaceutical companies with drugs used in affective and related disorders; is lead investigator for the Embolden Study (AZ), BCl Neuroplasticity study, and Aripiprazole Mania Study and investigator initiated studies from AZ, Eli Lilly, Lundbeck, and Wyeth; and has received grant funding from the National Institute of Mental Health (USA); Canadian Institutes of Health Research (Canada); National Alliance for Research on Schizophrenia and Depression (USA); Stanley Medical Research Institute (USA); Medical Research Council (UK); Wellcome Trust (UK); Royal College of Physicians (Edinburgh); British Medical Association (UK); University of British Columbia-Vancouver General Hospital Foundation (Canada); Western Economic Diversification Canada (Canada); Canadian Cardiovascular Society Depression Research Fund (Canada); Michael Smith Foundation for Health Research (Canada); and NIHR (UK). In the last 3 years RHMW has received support for research, expenses to attend conferences and fees for lecturing and consultancy work (including attending advisory boards) from various pharmaceutical companies including Astra Zeneca, Cyberonics, Eli Lilly, Janssen, Liva Nova, Lundbeck, MyTomorrows, Otsuka, Pfizer, Roche, Servier, SPIMACO and Sunovion.

RHMW, AHY and AJC all undertake clinical work within tertiary level specialist affective disorders services.

In 2015 AC was expert witness for a patent issue about quetiapine extended release.

AJC and AHY acknowledge support by the NIHR Biomedical Research Council (BRC). JG is an NIHR Senior Investigator and MJA and AC acknowledge support from the NIHR Oxford cognitive health Clinical Research Facility. The views expressed are those of the authors and not necessarily those of the NHS, the NIHR or the Department of Health. All other authors declare that they have no competing interests. All authors will have full access to the final trial dataset.

\section{Publisher's Note}

Springer Nature remains neutral with regard to jurisdictional claims in published maps and institutional affiliations.

\footnotetext{
Author details

${ }^{1}$ Centre for Affective Disorders, Department of Psychological Medicine, Institute of Psychiatry, Psychology \& Neuroscience, King's College London, London, UK. ${ }^{2}$ South London and Maudsley NHS Foundation Trust, London, UK. ${ }^{3}$ Biostatistics \& Health Informatics Department, Institute of Psychiatry, Psychology \& Neuroscience, King's College London, London, UK. ${ }^{4}$ Health Services and Population Research, Institute of Psychiatry, Psychology \& Neuroscience, King's College London, London, UK. ${ }^{5}$ Northumberland, Tyne and Wear NHS Foundation Trust, Newcastle upon Tyne, UK. Institute of Neuroscience, Newcastle University, Newcastle upon Tyne, UK. ${ }^{7}$ Lonsdale Medical Centre, London, UK. ${ }^{8}$ Department of Psychiatry, University of Oxford, Oxford, UK. ${ }^{9}$ Oxford Health NHS Foundation Trust, Oxford, UK. ${ }^{10}$ Tees, Esk and Wear Valleys NHS Foundation Trust, Darlington, UK.
} 
Received: 9 June 2017 Accepted: 21 June 2017

Published online: 26 June 2017

\section{References}

1. Kessler RC, Berglund P, Demler O, Jin R, Koretz D, Merikangas KR, et al. The epidemiology of major depressive disorder: results from the National Comorbidity Survey Replication (NCS-R). JAMA. 2003;289:3095-105.

2. Ustün TB, Ayuso-Mateos JL, Chatterji S, Mathers C, Murray CJL. Global burden of depressive disorders in the year 2000. Br J Psychiatry J Ment Sci. 2004;184:386-92.

3. Cleare A, Pariante CM, Young AH, Anderson IM, Christmas D, Cowen PJ, et al. Evidence-based guidelines for treating depressive disorders with antidepressants: a revision of the 2008 British Association for Psychopharmacology guidelines. J Psychopharmacol Oxf Engl. 2015;29:459525.

4. Rush AJ, Trivedi MH, Wisniewski SR, Nierenberg AA, Stewart JW, Warden D, et al. Acute and longer-term outcomes in depressed outpatients requiring one or several treatment steps: a STAR*D report. Am J Psychiatry. 2006;163: 1905-17.

5. Trivedi MH, Rush AJ, Wisniewski SR, Nierenberg AA, Warden D, Ritz L, et al. Evaluation of outcomes with citalopram for depression using measurementbased care in $\operatorname{STAR}^{*} \mathrm{D}$ : implications for clinical practice. Am J Psychiatry. 2006;163:28-40.

6. Wooderson SC, Fekadu A, Markopoulou K, Rane LJ, Poon L, Juruena MF, et al. Long-term symptomatic and functional outcome following an intensive inpatient multidisciplinary intervention for treatment-resistant affective disorders. J Affect Disord. 2014;166:334-42

7. Thase ME, Shelton RC, Khan A. Treatment with venlafaxine extended release after SSRI nonresponse or intolerance: a randomized comparison of standard- and higher-dosing strategies. J Clin Psychopharmacol. 2006;26: 250-8.

8. Fekadu A, Wooderson SC, Markopoulo K, Donaldson C, Papadopoulos A, Cleare AJ. What happens to patients with treatment-resistant depression? A systematic review of medium to long term outcome studies. J Affect Disord. 2009;116:4-11.

9. Wade AG, Crawford GM, Yellowlees A. Efficacy, safety and tolerability of escitalopram in doses up to $50 \mathrm{mg}$ in major depressive disorder (MDD): an open-label, pilot study. BMC Psychiatry. 2011;11:42.

10. Rudolph RL, Fabre LF, Feighner JP, Rickels K, Entsuah R, Derivan AT. A randomized, placebo-controlled, dose-response trial of venlafaxine hydrochloride in the treatment of major depression. J Clin Psychiatry. 1998; 59:116-22.

11. Burke WJ, Bose A. Fixed-dose trial of the single isomer SSRI escitalopram in depressed outpatients. J Clin Psychiatry. 2002;63:331-6.

12. Adli M, Baethge C, Heinz A, Langlitz N, Bauer M. Is dose escalation of antidepressants a rational strategy after a medium-dose treatment has failed? Eur Arch Psychiatry Clin Neurosci. 2005;255:387-400.

13. Dornseif BE, Dunlop SR, Potvin JH, Wernicke JF. Effect of dose escalation after low-dose fluoxetine therapy. Psychopharmacol Bull. 1989;25:71-9.

14. Licht RW, Qvitzau S. Treatment strategies in patients with major depression not responding to first-line sertraline treatment. A randomised study of extended duration of treatment, dose increase or mianserin augmentation. Psychopharmacology (Berl). 2002;161:143-51.

15. Schweizer E, Rynn M, Mandos LA, Demartinis N, García-España F, Rickels K. The antidepressant effect of sertraline is not enhanced by dose titration: results from an outpatient clinical trial. Int Clin Psychopharmacol. 2001;16:137-43.

16. Rush AJ, Trivedi MH, Stewart JW, Nierenberg AA, Fava M, Kurian BT, et al. Combining medications to enhance depression outcomes (CO-MED): acute and long-term outcomes of a single-blind randomized study. Am J Psychiatry. 2011;168:689-701.

17. Nelson JC, Papakostas Gl. Atypical antipsychotic augmentation in major depressive disorder: a meta-analysis of placebo-controlled randomized trials. Focus. 2010:8:570-82.

18. Bauer M, Adli M, Ricken R, Severus E, Pilhatsch M. Role of lithium augmentation in the management of major depressive disorder. CNS Drugs. 2014:28:331-42.

19. NICE. Depression in adults: recognition and management (Updated Edition). NICE Clin Guidel No 90. 2009. https://www.nice.org.uk/guidance/cg90/ chapter/1-Guidance.

20. Bauer M, Dell'osso L, Kasper S, Pitchot W, Dencker Vansvik E, Köhler J, et al. Extended-release quetiapine fumarate (quetiapine XR) monotherapy and quetiapine XR or lithium as add-on to antidepressants in patients with treatment-resistant major depressive disorder. J Affect Disord. 2013;151:209-19.

21. Dorée J-P, Des Rosiers J, Lew V, Gendron A, Elie R, Stip E, et al. Quetiapine augmentation of treatment-resistant depression: a comparison with lithium. Curr Med Res Opin. 2007;23:333-41.

22. Bauer M, Pfennig A, Severus E, Whybrow PC, Angst J, Möller H-J, et al. World Federation of Societies of biological psychiatry (WFSBP) guidelines for biological treatment of unipolar depressive disorders, part 1: update 2013 on the acute and continuation treatment of unipolar depressive disorders. World J Biol Psychiatry Off J World Fed Soc Biol Psychiatry. 2013;14:334-85.

23. Bauer M, Pretorius HW, Constant EL, Earley WR, Szamosi J, Brecher M. Extended-release quetiapine as adjunct to an antidepressant in patients with major depressive disorder: results of a randomized, placebo-controlled, double-blind study. J Clin Psychiatry. 2009;70:540-9.

24. Bauer M, Demyttenaere K, El-Khalili N, Thase ME, Papakostas Gl, Szamosi J, et al. Pooled analysis of adjunct extended-release quetiapine fumarate in patients with major depressive disorder according to ongoing SSRI or SNRI treatment. Int Clin Psychopharmacol. 2014;29:16-25.

25. El-Khalili $\mathrm{N}$, Joyce $\mathrm{M}$, Atkinson S, Buynak RJ, Datto $\mathrm{C}$, Lindgren $\mathrm{P}$, et al. Extended-release quetiapine fumarate (quetiapine $X R$ ) as adjunctive therapy in major depressive disorder (MDD) in patients with an inadequate response to ongoing antidepressant treatment: a multicentre, randomized, double-blind, placebo-controlled study. Int J Neuropsychopharmacol. 2010; 13:917-32.

26. Spielmans Gl, Berman MI, Linardatos E, Rosenlicht NZ, Perry A, Tsai AC. Adjunctive atypical antipsychotic treatment for major depressive disorder: a meta-analysis of depression, quality of life, and safety outcomes. PLoS Med. 2013;10:e1001403.

27. Zhou X, Ravindran AV, Qin B, Del Giovane C, Li Q, Bauer M, et al. Comparative efficacy, acceptability, and tolerability of augmentation agents in treatment-resistant depression: systematic review and network metaanalysis. J Clin Psychiatry. 2015;76:e487-98.

28. Chan A-W, Tetzlaff JM, Altman DG, Laupacis A, Gøtzsche PC, Krleža-Jerić K, et al. SPIRIT 2013 statement: defining standard protocol items for clinical trials. Ann Intern Med. 2013;158:200.

29. American Psychiatric Association. Diagnostic and Statistical Manual of Mental Disorders. Fifth Edition. American Psychiatric Association; 2013. http://psychiatryonline.org/doi/book/10.1176/appi.books.9780890425596. Accessed 23 Nov 2016.

30. Sheehan DV, Lecrubier $Y$, Harnett $K$, Amorim $P$, Janavs J, Weiller $E$, et al. The Mini-International Neuropsychiatric Interview (M.I.N.I): The development and validation of a structured diagnostic psychiatric interview for DSM-IV and ICD-10. J Clin Psychiatry. 1998;59 Suppl 20:22-33.

31. Hamilton M. A rating scale for depression. J Neurol Neurosurg Psychiatry. 1960;23:56-62.

32. Fekadu A, Wooderson S, Donaldson C, Markopoulou K, Masterson B, Poon L, et al. A multidimensional tool to quantify treatment resistance in depression: the Maudsley staging method. J Clin Psychiatry. 2009;70:177-84.

33. Taylor D, Paton C, Kapur S. The Maudsley prescribing guidelines in psychiatry, 12th Edition. Wiley-Blackwell; 2015.

34. Joint Formulary Committee. British National Formulary. 70th ed. London: BMJ Group and Pharmaceutical Press; 2015.

35. Montgomery SA, Asberg M. A new depression scale designed to be sensitive to change. Br J Psychiatry. 1979;134:382-9.

36. Busner J, Targum SD. The clinical global Impressions scale. Psychiatry Edgmont. 2007:4:28-37.

37. Rush AJ, Trivedi MH, Ibrahim HM, Carmody TJ, Arnow B, Klein DN, et al. The 16-item quick inventory of depressive symptomatology (QIDS), clinician rating (QIDS-C), and self-report (QIDS-SR): a psychometric evaluation in patients with chronic major depression. Biol Psychiatry. 2003;54:573-83.

38. Brooks $R$, Rabin $R$, De Charro $F$. The measurement and valuation of health status using EQ-5D: a European perspective: evidence from the EuroQol BIOMED research Programme. Springer Science \& Business Media; 2013. http://www.springer.com/gb/book/9781402012143. Accessed 14 Feb 2017.

39. Mundt JC, Marks IM, Shear MK, Greist JM. The work and social Adjustment scale: a simple measure of impairment in functioning. Br J Psychiatry. 2002; 180:461-4.

40. Clatworthy J, Bowskill R, Parham R, Rank T, Scott J, Horne R. Understanding medication non-adherence in bipolar disorders using a necessity-concerns framework. J Affect Disord. 2009;116:51-5. 
41. Wisniewski SR, Rush AJ, Balasubramani GK, Trivedi MH, Nierenberg AA, Investigators STARD. Self-rated global measure of the frequency, intensity, and burden of side effects. J Psychiatr Pract. 2006;12:71-9.

42. Chisholm D, Knapp MRJ, Knudsen HC, Amaddeo F, Gaite L, Wijngaarden BV. Client socio-demographic and service Receipt inventory - European version : development of an instrument for international research. Br J Psychiatry. 2000; 177:s28-33.

43. Rush AJ, Gullion CM, Basco MR, Jarrett RB, Trivedi MH. The inventory of depressive symptomatology (IDS): psychometric properties. Psychol Med. 1996;26:477-86.

44. Germans S, Van Heck GL, Moran P, Hodiamont PPG. The self-report standardized Assessment of personality-abbreviated scale: preliminary results of a brief screening test for personality disorders. Personal Ment Health. 2008;2:70-6.

45. Forty L, Kelly M, Jones L, Jones I, Barnes E, Caesar S, et al. Reducing the hypomania checklist (HCL-32) to a 16-item version. J Affect Disord. 2010;124:351-6.

46. Harrison J. THINC-it: what is it? European Neuropsychopharmacology; 2016. p. Page $\mathbf{S 7 5 9 .}$

47. White $I R$, Thompson SG. Adjusting for partially missing baseline measurements in randomized trials. Stat Med. 2005;24:993-1007.

48. Benjamini $Y$, Hochberg $Y$. Controlling the false discovery rate: a practical and powerful approach to multiple testing. J R Stat Soc Ser B Methodol. 1995;57:289-300

49. White IR, Horton NJ, Carpenter J, Pocock SJ, others. Strategy for intention to treat analysis in randomised trials with missing outcome data Bmj 2011;342:d40.

50. National Institute for Clinical Excellence. Depression: management of depression in primary and secondary care. Clinical practice guideline No 23. Lond NICE. 2004.

51. Loudon K, Treweek S, Sullivan F, Donnan P, Thorpe KE, Zwarenstein M. The PRECIS-2 tool: designing trials that are fit for purpose. BMJ. 2015:350:h2147.

52. Geddes JR, Gardiner A, Rendell J, Voysey M, Tunbridge E, Hinds C, et al. Comparative evaluation of quetiapine plus lamotrigine combination versus quetiapine monotherapy (and folic acid versus placebo) in bipolar depression (CEQUEL): a $2 \times 2$ factorial randomised trial. Lancet Psychiatry. 2016;3:31-9.

\section{Submit your next manuscript to BioMed Central and we will help you at every step:}

- We accept pre-submission inquiries

- Our selector tool helps you to find the most relevant journal

- We provide round the clock customer support

- Convenient online submission

- Thorough peer review

- Inclusion in PubMed and all major indexing services

- Maximum visibility for your research

Submit your manuscript at www.biomedcentral.com/submit 